\title{
Swift observations of IGR J16479-4514 in outburst (Research Note)
}

\author{
E. Bozzo ${ }^{1,2}$, A. Giunta ${ }^{2,3}$, L. Stella ${ }^{2}$, M. Falanga ${ }^{4}$, G. Israel $^{2}$, and S. Campana ${ }^{5}$ \\ 1 ISDC, Geneva Observatory, University of Geneva, Chemin d'Ecogia 16, 1290 Versoix, Switzerland \\ 2 INAF - Osservatorio Astronomico di Roma, Via Frascati 3300044 Rome, Italy \\ e-mail: bozzo@oa-roma.inaf.it \\ Dipartimento di Fisica - Università di Roma Tor Vergata, via della Ricerca Scientifica 1, 00133 Rome, Italy \\ 4 International Space Science Institute, Hallerstrasse 6, 3012 Bern, Switzerland \\ 5 INAF Osservatorio Astronomico di Brera, via Emilio Bianchi 46, 23807 Merate (LC), Italy
}

Received 22 March 2009 / Accepted 29 May 2009

\section{ABSTRACT}

\begin{abstract}
Aims. The supergiant fast X-ray transient source IGR J16479-4514 was observed in outburst two times with Swift. Its quiescent state was investigated in-depth only once in 2008 through a relatively long pointed observation with XMM-Newton. The latter observation was taken about 1.7 days after the outburst in 2008, and showed an X-ray eclipse-like event, likely caused by the supergiant companion. At present, this is the only supergiant fast X-ray transient that displayed an evidence for an X-ray eclipse.

Methods. Here we carry out a comparison between the most recent outburst of IGR J16479-4514, caught by Swift on 29 January 2009 and those detected previously from this source.

Results. The decay from the outbursts in 2005, 2008 and 2009 presents many similarities, and suggests a common mechanism that modulates the mass accretion rate onto the neutron star in IGR J16479-4514.
\end{abstract}

Key words. X-rays: binaries - stars: binaries: eclipsing - stars: individual: IGR J16479-4514 - stars: neutron - X-rays: stars

\section{Introduction}

IGR J16479-4514 is one of the supergiant fast X-ray transients (SFXT), a subclass of high mass X-ray binaries (HMXBs) hosting an accreting neutron star (NS) and a supergiant companion (Sguera et al. 2006; Walter et al. 2006). These sources undergo sporadic outbursts (peak luminosities of $\sim 10^{36}-10^{37} \mathrm{erg} \mathrm{s}^{-1}$ ), with typical durations of few thousands of seconds, and remain in quiescence for most of the time, with a mean X-ray luminosity in the $\sim 10^{32}-10^{34} \mathrm{erg} \mathrm{s}^{-1}$ range. In order to explain the behaviour of the SFXT sources, a number of different scenarios have been proposed (in't Zand 2005; Walter \& Zurita Heras 2007; Negueruela et al. 2008; Sidoli et al. 2007; Bozzo et al. 2008a). All these models involve a NS accreting from the intense wind of its supergiant companion, and interpret the transitions from outburst to quiescence as being due to strong variations in the mass accretion rate onto the NS. These variations have been ascribed to a centrifugal and/or magnetic gating mechanism by the NS magnetosphere (Bozzo et al. 2008a), or to clumps and structures in the wind of the supergiant star (Walter \& Zurita Heras 2007; Negueruela et al. 2008). Sidoli et al. (2007) proposed that SFXT outbursts result from a neutron star in a tilted orbit crossing a dense equatorial disk around the supergiant companion. As different models make different predictions with respect to the origin of the quiescent emission in SFXTs, an indepth knowledge of the timing and spectral properties of the low activity states of these sources is expected to help discriminating between the above models (Bozzo et al. 2008a). At present, only a few observations of quiescent SFXTs have been carried out, and thus very little is known on the mechanism that triggers the transitions between outburst and quiescence.
IGR J16479-4514 was observed with Swift in outburst two times, on 2005 August 30 (Sguera et al. 2008) and 2008 March 19 (Romano et al. 2008a,b). Its X-ray emission properties during these bright events is fairly well known. On the contrary, the quiescent state of IGR J16479-4514 was investigated in-depth only once with XMM-Newton ${ }^{1}$. This observation lasted $\sim 32 \mathrm{ks}$ and was carried out about 1.7 days after the bright outburst in 2008 , with the aim of gaining insight on the mechanism that drive the quiescent emission of this source. Surprisingly, XMM-Newton caught IGR J16479-4514 undergoing a transition from a bright state $\left(2-10 \mathrm{keV}\right.$ absorbed flux $\left.1.0 \times 10^{-11} \mathrm{erg} / \mathrm{cm}^{2} / \mathrm{s}\right)$ to a lower flux state $\left(2-10 \mathrm{kev}\right.$ absorbed flux $\left.7.5 \times 10^{-13} \mathrm{erg} / \mathrm{cm}^{2} / \mathrm{s}\right)$. The spectral and timing analysis of this observation showed unambiguously that the transition was due to the obscuration by an occulting body, most likely an eclipse by the supergiant companion. This revealed that at least some of the very large X-ray luminosity swings displayed by the SFXT sources might not be due only to genuine changes in the mass accretion rate.

Here we report on the most recent outburst of IGR J16479-4514, detected by Swift on 2009 January 29, and compare this outburst with those observed previously in 2005 , and 2008.

\section{Data analysis and results}

Swift /BAT caught a new bright outburst from IGR J16479-4514 on 2009 January 29 (Romano et al. 2009). The start time of the

\footnotetext{
${ }^{1}$ Walter et al. (2006) also reported on a $\sim 2.9$ ks XMM-Newton observation of this source in quiescence, but it was affected by a very strong and variable background.
} 
Table 1. Observations log of IGRJ16479-4514 (see note at the bottom of the table for other specifications).

\begin{tabular}{|c|c|c|c|c|c|c|c|c|}
\hline $\begin{array}{l}\text { OBS ID } \\
(2009)\end{array}$ & INSTR & START TIME & STOP TIME & $\begin{array}{l}\text { EXP } \\
(s)\end{array}$ & $\begin{array}{l}N_{\mathrm{H}} \\
\left(10^{22} \mathrm{~cm}^{-2}\right)\end{array}$ & $\Gamma$ & $\begin{array}{l}F_{\mathrm{obs}} \\
\left(\mathrm{erg} / \mathrm{cm}^{2} / \mathrm{s}\right)\end{array}$ & $\begin{array}{l}\chi_{\text {red }}^{2} / \text { d.o.f. } \\
\text { (C-stat/d.o.f.) }\end{array}$ \\
\hline \multirow[t]{2}{*}{00341452000} & BAT & 2009-01-29 06:26:12 & 2009-01-29 07:56:43 & $1.6 \mathrm{E}+03$ & - & $2.5_{-0.3}^{+0.3}$ & $2.55 \times 10^{-9}$ & $0.9 / 27$ \\
\hline & XRT/PC & 2009-01-29 06:47:35 & 2009-01-29 08:39:04 & $2.6 \mathrm{E}+03$ & $4.7_{-1.1}^{+1.3}$ & $1.0_{-0.3}^{+0.3}$ & $1.0_{-0.2}^{+0.1} \times 10^{-10}$ & $1.1 / 44$ \\
\hline 00030296077 & $\mathrm{XRT} / \mathrm{PC}$ & 2009-01-29 15:59:44 & 2009-01-29 22:36:24 & $5.9 \mathrm{E}+03$ & $5.7_{-1.5}^{+1.1}$ & $1.6_{-0.4}^{+0.4}$ & $4.1_{-16}^{+0.2} \times 10^{-11}$ & $1.2 / 33$ \\
\hline 00030296078 & $\mathrm{XRT} / \mathrm{PC}$ & 2009-01-30 05:05:45 & 2009-01-30 07:01:56 & $2.5 \mathrm{E}+03$ & $9.3_{-3.2}^{+3.6}$ & $1.5_{-0.6}^{+0.7}$ & $3.1_{-1.8}^{+0.3} \times 10^{-11}$ & $1.2 / 21$ \\
\hline 00030296080 & $\mathrm{XRT} / \mathrm{PC}$ & 2009-02-01 19:50:46 & 2009-02-01 21:30:57 & $2.0 \mathrm{E}+03$ & 9.3 (fixed) & 1.5 (fixed) & $4.5_{-0.7}^{+0.9} \times 10^{-12}$ & - \\
\hline 00030296081 & $\mathrm{XRT} / \mathrm{PC}$ & 2009-02-02 08:46:12 & 2009-02-02 11:40:57 & $1.9 \mathrm{E}+03$ & 9.3 (fixed) & 1.5 (fixed) & $3.4_{-0.7}^{+0.7} \times 10^{-12}$ & - \\
\hline 00030296082 & $\mathrm{XRT} / \mathrm{PC}$ & 2009-02-03 18:25:48 & 2009-02-03 21:49:58 & $1.2 \mathrm{E}+03$ & $9.3(\text { fixed })^{a}$ & $1.5{\text { (fixed })^{a}}^{a}$ & $2.1 \times 10^{-12}$ & - \\
\hline 00030296083 & $\mathrm{XRT} / \mathrm{PC}$ & 2009-02-04 00:51:50 & 2009-02-04 04:09:56 & $1.4 \mathrm{E}+03$ & 9.3 (fixed) & 1.5 (fixed) & $1.1_{-0.5}^{+0.5} \times 10^{-12}$ & - \\
\hline 00030296084 & $\mathrm{XRT} / \mathrm{PC}$ & 2009-02-05 02:24:25 & 2009-02-05 02:50:30 & $1.5 \mathrm{E}+03$ & 9.3 (fixed) & 1.5 (fixed) & $1.2_{-0.2}^{+0.2} \times 10^{-11}$ & - \\
\hline 00030296085 & XRT/PC & 2009-02-06 01:13:12 & 2009-02-06 04:28:57 & $4.7 \mathrm{E}+02$ & 9.3 (fixed) & 1.5 (fixed) & $1.5_{-0.3}^{+0.3} \times 10^{-11}$ & - \\
\hline 00030296087 & $\mathrm{XRT} / \mathrm{PC}$ & 2009-02-08 18:54:08 & 2009-02-08 22:15:57 & $1.6 \mathrm{E}+03$ & $6.9_{-2.4}^{+2.7}$ & $1.1_{-0.5}^{+0.6}$ & $1.7_{-0.1}^{+0.1} \times 10^{-10}$ & $0.9 / 26$ \\
\hline 00030296088 & $\mathrm{XRT} / \mathrm{PC}$ & 2009-02-10 03:10:18 & 2009-02-10 08:06:57 & $1.5 \mathrm{E}+03$ & $6.9{(\text { fixed })^{a}}^{a}$ & 1.2 (fixed) $^{a}$ & $1.6 \times 10^{-12}$ & - \\
\hline 00030296090 & XRT/PC & 2009-02-12 22:30:18 & 2009-02-13 00:20:56 & $1.7 \mathrm{E}+03$ & 10.6 (fixed) & 2.1 (fixed) & $1.1_{-0.4}^{+0.4} \times 10^{-12}$ & - \\
\hline 00030296091 & $\mathrm{XRT} / \mathrm{PC}$ & 2009-02-13 05:04:18 & $2009-02-13$ 06:45:54 & $1.3 \mathrm{E}+03$ & 10.6 (fixed) & 2.1 (fixed) & $1.1_{-0.5}^{+0.5} \times 10^{-12}$ & - \\
\hline 00030296092 & $\mathrm{XRT} / \mathrm{PC}$ & 2009-02-14 06:34:01 & 2009-02-14 08:18:56 & $1.3 \mathrm{E}+03$ & $8.3_{-5.2}^{+6.8}$ & $1.0_{-1.1}^{+1.2}$ & $2.0_{-1.4}^{+0.5} \times 10^{-11}$ & $(26.46 / 24)^{b}$ \\
\hline 00030296093 & $\mathrm{XRT} / \mathrm{PC}$ & 2009-02-15 03:19:41 & 2009-02-15 03:39:57 & $1.2 \mathrm{E}+03$ & 8.3 (fixed) & 1.0 (fixed) & $4.0_{-0.9}^{+0.9} \times 10^{-12}$ & - \\
\hline 00030296094 & $\mathrm{XRT} / \mathrm{PC}$ & 2009-02-16 08:26:29 & 2009-02-16 10:14:42 & $1.1 \mathrm{E}+03$ & $11.1_{-4.3}^{+6.0}$ & $2.1_{-1.0}^{+1.1}$ & $1.9_{-1.4}^{+0.3} \times 10^{-11}$ & $(23.41 / 29)^{b}$ \\
\hline 00030296095 & $\mathrm{XRT} / \mathrm{PC}$ & 2009-02-17 00:22:55 & 2009-02-17 00:38:50 & $9.5 \mathrm{E}+02$ & 11.1 (fixed) $^{a}$ & 2.1 (fixed) $^{a}$ & $1.3 \times 10^{-12}$ & - \\
\hline $\begin{array}{l}\text { OBS ID } \\
(2008)\end{array}$ & INSTR & START TIME & STOP TIME & $\begin{array}{l}\text { EXP } \\
(s)\end{array}$ & $\begin{array}{l}N_{\mathrm{H}} \\
\left(10^{22} \mathrm{~cm}^{-2}\right)\end{array}$ & $\Gamma$ & $\begin{array}{l}F_{\mathrm{obs}} \\
\left(\mathrm{erg} / \mathrm{cm}^{2} / \mathrm{s}\right)\end{array}$ & $\begin{array}{l}\chi_{\text {red }}^{2} / \text { d.o.f. } \\
\text { (C-stat/d.o.f.) }\end{array}$ \\
\hline \multirow[t]{3}{*}{00306829000} & BAT & 2008-03-19 22:42:48 & 2008-03-19 22:56:50 & $6.3 \mathrm{E}+02$ & - & $2.8_{-0.1}^{+0.1}$ & $6.6 \times 10^{-9}$ & $1.5 / 27$ \\
\hline & XRT/WT & 2008-03-19 22:46:47 & 2008-03-19 23:55:26 & $9.1 \mathrm{E}+02$ & $6.0_{-0.3}^{+0.3}$ & $1.1_{-0.1}^{+0.1}$ & $4.4_{-0.1}^{+0.1} \times 10^{-9}$ & $1.1 / 284$ \\
\hline & XRT/PC & 2008-03-19 23:55:27 & 2008-03-20 00:00:53 & $3.2 \mathrm{E}+02$ & 6.0 (fixed) & 1.1 (fixed) & $6.7_{-0.9}^{+0.9} \times 10^{-11}$ & - \\
\hline 00030296030 & $\mathrm{XRT} / \mathrm{PC}$ & 2008-03-21 02:05:29 & 2008-03-21 03:48:58 & $8.1 \mathrm{E}+02$ & 6.0 (fixed) & 1.1 (fixed) & $8.9_{-1.9}^{+1.9} \times 10^{-12}$ & - \\
\hline 00030296031 & $\mathrm{XRT} / \mathrm{PC}$ & 2008-03-21 21:22:28 & 2008-03-21 23:05:57 & $8.2 \mathrm{E}+02$ & 6.0 (fixed) & 1.1 (fixed) & $4.7_{-3.7}^{+3.3} \times 10^{-13}$ & - \\
\hline 00030296032 & XRT/PC & $2008-03-23$ 05:25:03 & $2008-03-23$ 05:35:30 & $6.0 \mathrm{E}+02$ & 6.0 (fixed) & 1.1 (fixed) & $3.5_{-1.1}^{+1: 1} \times 10^{-12}$ & - \\
\hline 00030296033 & $\mathrm{XRT} / \mathrm{PC}$ & 2008-03-24 05:36:51 & 2008-03-24 06:50:56 & $1.0 \mathrm{E}+03$ & $12.2_{-7.5}^{+8.8}$ & $1.7_{-1.2}^{+1.3}$ & $2.6_{-2.0}^{+0.1} \times 10^{-11}$ & $(30.9 / 37)^{b}$ \\
\hline $\begin{array}{l}\text { OBS ID } \\
(2005)\end{array}$ & INSTR & START TIME & STOP TIME & $\begin{array}{l}\text { EXP } \\
(s)\end{array}$ & $\begin{array}{l}N_{\mathrm{H}} \\
\left(10^{22} \mathrm{~cm}^{-2}\right)\end{array}$ & $\Gamma$ & $\begin{array}{l}F_{\text {obs }} \\
\left(\mathrm{erg} / \mathrm{cm}^{2} / \mathrm{s}\right)\end{array}$ & $\begin{array}{l}\chi_{\text {red }}^{2} / \text { d.o.f. } \\
\text { (C-stat/d.o.f. })\end{array}$ \\
\hline 00152652000 & BAT & 2005-08-30 04:03:49 & 2005-08-30 04:13:51 & $6.3 \mathrm{E}+02$ & - & $2.0_{-0.7}^{+0.7}$ & $2.7 \times 10^{-9 c}$ & $1.2 / 14$ \\
\hline \multirow[t]{2}{*}{00030296001} & XRT/PC & 2005-08-30 04:12:41 & 2005-09-01 14:01:58 & $1.1 \mathrm{E}+04$ & $6.3_{-1.2}^{+1.4}$ & $1.0_{-0.3}^{+0.3}$ & $3.3_{-2.2}^{+0.3} \times 10^{-11}$ & $0.9 / 44$ \\
\hline & XRT/WT & 2005-08-30 04:11:00 & 2005-09-01 13:40:51 & $4.5 \mathrm{E}+03$ & $6.0_{-2.0}^{+3.0}$ & $1.2_{-0.5}^{+0.6}$ & $2.2_{-0.2}^{+0.3} \times 10^{-11}$ & $0.9 / 33$ \\
\hline 00030296002 & XRT/PC & 2005-09-10 00:15:00 & 2005-09-12 12:10:59 & $6.3 \mathrm{E}+03$ & $9.7_{-2.8}^{+3.6}$ & $1.2_{-0.5}^{+0.6}$ & $5.2_{-3.0}^{+0.2} \times 10^{-11}$ & $1.9 / 27$ \\
\hline 00030296003 & XRT/PC & 2005-09-14 00:43:34 & 2005-09-14 10:47:57 & $4.2 \mathrm{E}+03$ & 9.7 (fixed) & 1.2 (fixed) & $5.7_{-10}^{+1.0} \times 10^{-13}$ & - \\
\hline
\end{tabular}

Note: spectra extracted from these observations are fit with an absorbed power law (absorption column density $N_{\mathrm{H}}$ and photon index $\Gamma$ ). $F_{\text {obs }}$ is the. XRT/PC or XRT/WT (BAT) absorbed flux in the $0.3-10 \mathrm{keV}(15-80 \mathrm{keV})$ energy band. EXP indicates the total exposure time of each observation.

(Swift observations comprise several snapshots and are not continuous pointings at the source). ${ }^{a} 3 \sigma$ upper limit. ${ }^{b} \mathrm{C}$-statistic has been used to fit the spectrum. ${ }^{c}$ In this case the BAT flux is in the energy range $15-50 \mathrm{keV}$.

BAT trigger was 06:33:07 UTC (including UTCF correction). Swift /XRT slewed to the source about 800 s after the BAT trigger, and observed in Windowed Timing (WT) mode during the first $46 \mathrm{~s}$. Data in photon counting (PC) mode were then accumulated for a total exposure time of $2.6 \mathrm{ks}$. We analyzed the data from observation ID. 00341452000, that includes the BAT data of the outburst, as well as the following XRT WT and PC data, and use also Swift follow-up observations that were carried out up to 20 days after the BAT trigger.

In order to compare the 2009 outburst with those detected previously, we also analyzed Swift observations of this source that were carried out during the outbursts in 2005 and 2008 (these observations were previously published by Sguera et al. 2008; Romano et al. 2008a). We used outburst and follow-up observations up to 20 days after each BAT trigger. A log of the data sets is given in Table 1 .

The Swift data were analyzed by using standard procedures (Burrows et al. 2005) and the latest calibration files available.
The BAT and XRT data were processed with the BATPRODUCT (v.2.42) and XRTPIPELINE (v.0.12.1) tasks, respectively. Filtering and screening criteria were applied by using FTOOLS (Heasoft v.6.6.1). We extracted source and background light curves and spectra by selecting event grades of $0-2$ and $0-12$, respectively for the WT and PC mode. Exposure maps were created through the XRTEXPOMAP task, and we used the latest spectral redistribution matrices in the HEASARC calibration database (v.011). Ancillary response files, accounting for different extraction regions, vignetting and PSF corrections, were generated by using the XRTMKARF task. When required, we corrected PC data for pile-up, and used the XRTLCCORR to account for this correction in the background-subtracted light curves.

For each observation in Table 1, we extracted the light curve and spectrum, and derived a mean X-ray flux by fitting this spectrum with an absorbed power law model (we used XSPEC v.12.5.0). Spectra with an adequate statistics were rebinned in order to have at least 20 photons per bin so as to permit $\chi^{2}$ fitting. 


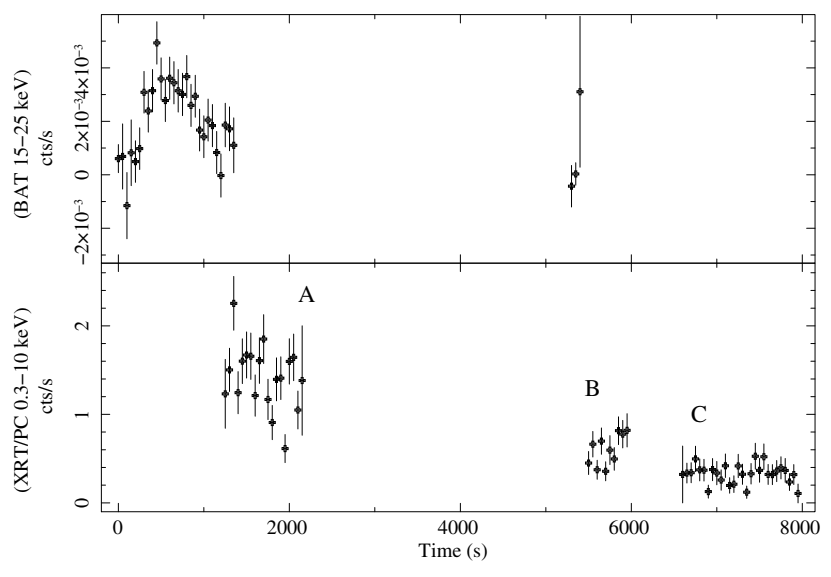

Start Time 14860 6:27:42:184 Stop Time 14860 8:40:12:184

Fig. 1. BAT and XRT/PC light curve of the 2009 outburst (time bin is $50 \mathrm{~s})$.

Lower statistic spectra were rebinned in order to have at least 5 photons per bin and then fit by using the C-statistics (Cash 1979). Observations with less than 100 counts were treated separately, as the $\mathrm{C}$-statistics is not recommended with such a small number of photons ${ }^{2}$. In these cases we estimated the source count rate of the observation with SOSTA (XIMAGE V.4.4.1), and then used this count rate within WEBPIMMS ${ }^{3}$ in order to derive the X-ray flux (we assumed the same spectral model of the closest observation for which a spectral analysis could be carried out).

Figure 1 shows the BAT $(15-25 \mathrm{keV})$ and XRT/PC (0.3-10 keV) light curve of the 2009 outburst (XRT/WT data in the $0.3-10 \mathrm{keV}$ energy band are included only in Fig. 4). As the observation interval of BAT and XRT/PC do not significantly overlap, we did not attempt to fit a combined BAT+XRT spectrum. Instead we fit them separately and report the results of these fits in Table 1. The BAT spectrum, integrated over the total duration of the outburst, and the XRT/PC spectrum of observation ID $00341452000(\mathrm{~A}+\mathrm{B}+\mathrm{C}$ intervals $)$ are shown in Fig. 2, together with the best fits and the residuals from these fits. We also searched for spectral variability by extracting time resolved spectra in the intervals $\mathrm{A}, \mathrm{B}$, and $\mathrm{C}$ of Fig. 1. This analysis revealed that the source $\mathrm{X}$-ray flux decreased from $3.2 \times 10^{-10} \mathrm{erg} / \mathrm{cm}^{2} / \mathrm{s}$ in interval A, to $3.6 \times 10^{-11} \mathrm{erg} / \mathrm{cm}^{2} / \mathrm{s}$ in interval $\mathrm{C}$. The best fit powerlaw index and the absorption column density did not show any significant variation (they were all constant within the errors).

In Fig. 3 we plot the evolution (up to $\sim 20$ days from the BAT trigger) of the absorbed $2-10 \mathrm{keV}$ X-ray flux during the 2005, 2008 and 2009 outburst of IGR J16479-4514. In this figure we used the fluxes from Table 1 and considered half of each observation duration as the error on the time axis.

Finally, in Fig. 4 we show the combined XRT/PC and XRT/WT light curves of the three outburts in detail. For clarity, we divided the count rate of the first 5 points (XRT/WT data) of the 2008 light curve by a factor of 10 .

It is seen from Fig. 1 that the rise and decay times of the 2009 outburst are of order of a few thousand seconds, similar to the time scales measured in the 2005 and 2008 outbursts (Sguera et al. 2008; Romano et al. 2008a). The fluxes and spectral properties of the source emission during the peak and the beginning of the outburst decay (up to $\sim 7 \mathrm{ks}$ after the BAT trigger) are

\footnotetext{
2 https://astrophysics.gsfc.nasa.gov/XSPECwiki/

statistical_methods_in_XSPEC

${ }^{3}$ http://heasarc.nasa.gov/Tools/w3pimms.html
}

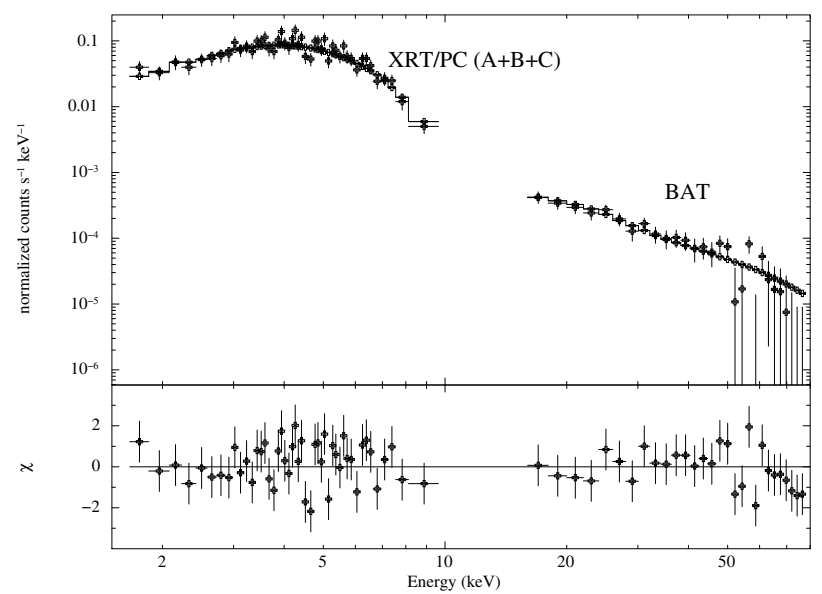

Fig. 2. BAT and XRT/PC spectra of the 2009 outburst. The BAT spectrum is integrated over the total outburst duration, whereas the XRT/PC spectrum is integrated over the $\mathrm{A}+\mathrm{B}+\mathrm{C}$ time intervals shown in Fig. 1. The former spectrum was fit by using a simple power law, whereas for the latter we also included the effect of the absorption at low energies (the best fit parameters are reported in Table 1). The lower panel shows the residuals from the best fits.
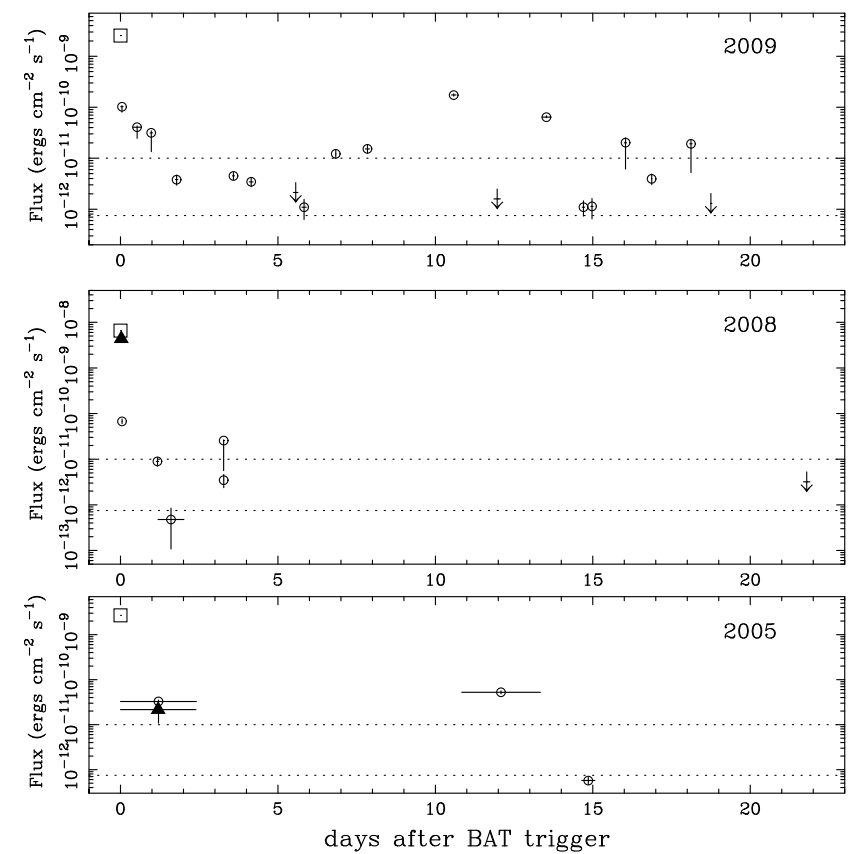

Fig. 3. Evolution of the X-ray flux during the 2005, 2008, and 2009 outbursts. We used all the observations reported in Table 1, and considered the BAT trigger time as the origin of the $X$ axis. The BAT trigger times are 2005-08-30 04:08:48 UTC, 2008-03-19 22:44:45 UTC, and 2009-01-29 06:33:07 UTC. In all panels, squares indicates the BAT X-ray flux (15-80 keV for the 2009 and 2008 outburst and 15-50 keV for the 2005 outburst; we did not indicated the errors for these fluxes), open circles and triangles are for XRT/PC and XRT/WT fluxes (absorbed flux in the $2-10 \mathrm{keV}$ band), respectively. The arrows indicate $3 \sigma$ upper limits. Errors on the $X$ axis correspond to the half duration of each observation.

also in agreement with previously reported results. The typical outburst spectrum at high energy $(15-50 \mathrm{keV})$ can be fit by a power law of photon index $\sim 2.5$. The mean $\mathrm{X}$-ray flux is of few times $10^{-9} \mathrm{erg} / \mathrm{cm}^{2} / \mathrm{s}$ (Sguera et al. 2008). The $2-10 \mathrm{keV}$ spectrum is flatter and can be well fit by using a power law of photon index $\Gamma \sim 1$ and an absorption column density of 

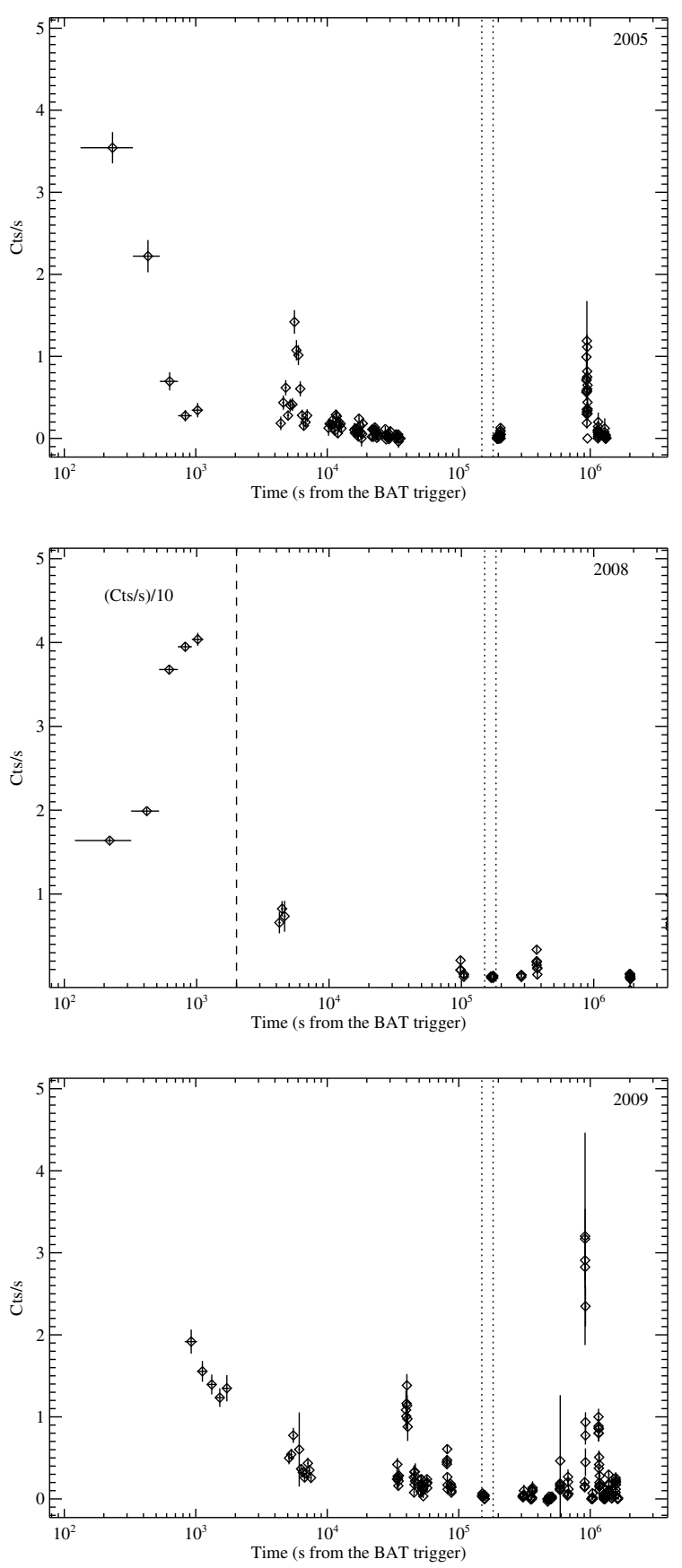

Fig. 4. XRT/PC and XRT/WT light curves of the three outbursts in 2005, 2008, and 2009 (time bin is $200 \mathrm{~s}$ ). All the reported times are measured from the BAT triggers (see the caption of Fig. 3). In the light curve of the 2008 outburst, the beginning and the end of the XMM-Newton observation that could have indicated an X-ray eclipse are marked with dotted vertical lines. In the light curves of the other two outbursts, the dotted lines indicate where the suspected eclipse should have been observed. For clarity, we divided the count rate of the first 5 points (XRT/WT data) of the 2008 light curve by a factor of 10 (these points are separated from the others through a dashed line).

$\sim 5 \times 10^{22} \mathrm{~cm}^{-2}$ (Romano et al. 2008a). We did not attempt to fit the broad-band spectrum of the 2009 outburst, since the observation intervals of BAT and XRT do not overlap significantly (see Fig. 1). Moreover, owing to poor statistics of the XRT/PC data, time resolved spectra could not be investigated during the first $7 \mathrm{ks}$ after the BAT trigger (intervals A, B and C in Fig. 1).
Therefore, we could not search for spectral variations during the decay from the outburst. However, we note that follow up observations carried out up to 20 days after the BAT trigger showed a moderate increase of the absorption column density (a factor of $\sim 2$ ) and a steepening of the power law index (from $\sim 1$ to $\sim 2$, see Table 1).

In order to compare the 2009 outburst with those detected previously in 2005 and 2008, we studied the variation of the X-ray flux from IGR J16479-4514 during the 20 days that followed each of these outbursts. This is shown in Fig. 3. In the middle panel of this figure we give the X-ray flux of the 2008 outburst; the horizontal dotted lines represent the high and lower flux state of IGR J16479-4514 as observed in 2008 by XMMNewton (see Sect. 1). According to the results discussed in Bozzo et al. (2008b), these correspond to the average X-ray flux during the eclipse ingress and the mid-eclipse, respectively. For comparison, these two dotted lines are also plotted in the upper and lower panels of Fig. 3, that give the evolution of the X-ray flux during the 2009 and 2005 outbursts, respectively. We note that, in all the three cases, several observations have an X-ray flux that falls inside the range defined by the two dotted lines. As a consequence of this and the relatively sparse time coverage, the plots in Fig. 3 do not permit to single out the presence of an X-ray eclipse. Rather IGR J16479-4514 may simply alternate between higher and lower flux states; unfortunately, the origin of these states can hardly be investigated without a detailed spectral analysis ${ }^{4}$.

By setting the start times of all light curves in Fig. 3 at the onset of the outbursts, we can compare the flux evolution and, in particular, verify whether the eclipse-like event $\sim 1.7 \mathrm{~d}$ after the 2008 event, has a counterpart in the 2005 and 2009 light curves for comparable delays. Stated differently, this amounts to checking whether the outburst and the eclipse-like event are driven by the same, possibly orbital, "clock".

Swift observations in 2008 clearly show that the source X-ray flux between 1-2 days after the BAT trigger was compatible with the lower flux measured by XMM-Newton (i.e. the mid-eclipse state). Furthermore, the mean X-ray flux of the subsequent observation in 2008 (that is compatible with the eclipse egress, if the eclipse is symmetric), suggests an upper limit to the duration the eclipse-like event of about $\sim 3$ days. Even thought the orbital period of IGR J16479-4514 is still not known, this limit seems reasonable, as in other SFXTs an orbital period of $\sim 30$ days has been measured (Bird et al. 2009; Zurita-Heras \& Chaty 2009), and, of course, an eclipse can last up to half of the orbital period. However, if we supposed that the eclipse-like event takes place at a fixed delay from the beginning of the outburst, then the 2009 observation would indicate that the eclipse duration is shorter. In fact, from the upper panel of Fig. 3 we note that the source was observed at an X-ray flux comparable with the eclipse egress about $\sim 2$ days after the BAT trigger, thus limiting the eclipse duration to less than one day. A comparison with the 2005 outburst is more complicated because the XRT observation carried out after the BAT trigger has a large gap close to the expected eclipse ingress and egress (see below).

In order to further study the source behavior during the 2005 , 2008, and 2009 outbursts, we also investigated in detail the $\mathrm{X}$-ray light curves of all observations in Table 1 . This is shown in Fig. 4 (times on the $X$ axis are measured from the start time of the BAT trigger). In the middle panel of this figure we represented

4 The 2008 XMM-Newton spectra, on the contrary, provided convincing evidence that the lower intensity state resulted from an occultation episode. 
with dotted vertical lines the time interval in which the 2008 eclipse-like event was seen. Under the hypothesis that the X-ray eclipse of IGR J16479-4514 always follows the outburst, the two dotted lines in the upper and lower panel of Fig. 4 correspond to the position where an eclipse should have been observed after the outbursts in 2009 and 2005, respectively. Even if the poor statistics of the Swift data did not permit a detailed spectral analysis, and thus did not provide further support in favour of this hypothesis, we note that the source count-rate at these positions is consistent within all the three outbursts.

Apart from the X-ray eclipse-like event, we also note from Fig. 3 that the three outbursts displayed many other similarities. Particularly interesting is the fact the source showed in all the reported outbursts an increase in the X-ray activity after about $6 \mathrm{ks}$ from the BAT trigger (note that, close to this time interval the higher source count rate triggered XRT observations in WT mode in all three cases). Furthermore, we note the presence of a bright flare (XRT/PC count rate $>1$ cts/s) about $\sim 10$ days after the BAT triggers in 2009 and 2005 (see also, La Parola et al. 2009). The presence of this flare could not be investigated during the outburst in 2008, because no X-ray observation of IGR J16479-4514 was carried out at this period.

\section{Discussion and conclusion}

The similarities we pointed out in the post-outburst decay of IGR J16479-4514 in 2005, 2008 and 2009 suggest the presence of a stable structure in the system (at least for a few years), that is able to modulate the mass accretion rate onto the NS in a fairly reproducible fashion and gives rise to the observed X-ray activity. At present, it is not clear if other SFXT display a similar post-outburst behaviour. If this was the case, the implications for SFXT models would have to be assessed.

After this paper was submitted for publication, Jain et al. (2009) reported the discovery of $0.6 \mathrm{~d}$ long X-ray eclipse and a $3.3194 \mathrm{~d}$ orbital period in the Swift/BAT data of IGRJ164794514. These results, besides confirming the interpretation by Bozzo et al. (2008a) that the sudden drop-off in the source X-ray flux detected by XMM-Newton in 2008 was due to an $\mathrm{X}$-ray eclipse, provided also a clear confirmation of the conjecture proposed in the present research note, namely that the source outbursts of 2005, 2008 and 2009 preceded the X-ray eclipse (in 2008) and the lowest X-ray fluxes intervals (in 2005 and 2009) by the same amount of time. Indeed by using the orbital period and eclipse duration of Jain et al. (2009) and the eclipse onset time from the XMM-Newton observation of 2008 plus $0.3 \mathrm{~d}$ to derive the eclipse center time (leading to a superior conjunction epoch of MJD 54 547, 05 418, which we adopt as orbital phase 0 ), we verified that the onset of the 2005, 2008, and 2009 outbursts, as measured by the $S$ wift/BAT trigger time, occurred at an orbital phase of $0.36 \pm 0.09,0.3654 \pm 0.0002$, and $0.36 \pm 0.03$ respectively. Moreover the $0.6 \mathrm{~d}$ eclipse duration determined by Jain et al. (2009) agrees well with what we suggested in Sect. 2 based on the Swift/XRT data of the 2009 outburst.

These findings provide strong evidence that the source outbursts, though rare, take place around an orbital phase of $\sim 0.4$ and are thus inherently connected to the orbital motion, though the conditions that lead to their occurrence are met only rarely. Outbursts recurring at the orbital period were observed in three other SFXTs (SAX J1818.6-1703, IGR J11215-5952, and IGR J18483-0311; Bird et al. 2009; Zurita-Heras \& Chaty 2009; Sidoli et al. 2007; Sguera et al. 2007), and are reminiscent of type I outbursts in Be star X-ray transients. These episodes of intense accretion are likely related to periastron passage, where the supergiants' wind density is higher and its velocity relative to the neutron star lower (Stella et al. 1986). Alternatively the outbursts might be due to the neutron star crossing an equatorial dense disk ejected by the supergiant (Sidoli et al. 2007).

Acknowledgements. We thank the anonymous referee for his/her helpful comments, and the Swift staff, for having carried out ToO and follow-up observations of IGR J16479-4514. EB thanks M. Perri, M. Capalbi, and K. Page for their support during the Swift/XRT and Swift/BAT data analysis. This work was partially supported through ASI and MIUR grants.

\section{References}

Bird, A. J., Bazzano, A., Hill, A. B., et al. 2009, MNRAS, 393, L11

Bozzo, E., Falanga, M., \& Stella, L. 2008a, ApJ, 683, 1031

Bozzo, E., Stella, L., Israel, G., Falanga, M., \& Campana, S. 2008b, MNRAS, 391, L108

Burrows, D. N., Hill, J. E., Nousek, J. A., et al. 2005, Space Sci. Rev., 120, 165 Cash, W. 1979, ApJ, 228, 939

in 't Zand 2005, A\&A, 441, L1

Chetana, J., Biswajit, P., \& Anjan, D. 2009, MNRAS, in press [arXiv:0903.5403]

Jansen, A., Lumb, D., Altieri, B., et al. 2001, A\&A, 365, L1

La Parola, V., et al. 2009, Astr. Tel., 1929

Negueruela, I., Torrejon, J. M., Reig, P., Ribo, M., \& Smith, D. M. 2008, AIPC, 1010, 252

Rahoui, F., Chaty, S., Lagage, P., \& Pantin, E. 2008, A\&A, 484, 801

Romano, P., Sidoli, L., Mangano, V., et al. 2008a, ApJ, 680, L137

Romano, P., et al. 2008b, Astr. Tel., 1435

Romano, P., et al. 2009, Astr. Tel., 1920

Sguera, V., Bazzano, A., Bird, A. J., et al. 2006, ApJ, 646, 452

Sguera, V., Hill, A. B., Bird, A. J., et al. 2007, A\&A, 467, 249

Sguera, V., Bassani, L., Landi, R., et al. 2008, A\&A, 487, 619

Sidoli, L., Romano, P., Mereghetti, S., et al. 2007, A\&A, 476, 1307

Stella, L., White, N. E., \& Rosner, R. 1986, ApJ, 308, 669

Walter, R., Zurita, H. J., Bassani, L., et al. 2006, A\&A, 453, 133

Walter, R., \& Zurita Heras, J. A. 2007, A\&A, 476, 335

Zurita Heras, J. A., \& Chaty, S. 2009, A\&A, 493, L1 